\title{
BMJ Open Improving care for victims: a study protocol of the evaluation of a centre for sexual and family violence
}

\author{
E Zijlstra, ${ }^{1}$ S LoFoWong, ${ }^{1}$ G Hutschemaekers, ${ }^{2}$ A Lagro-Janssen ${ }^{1}$
}

To cite: Zijlstra E,

LoFoWong $\mathrm{S}$,

Hutschemaekers G, et al. Improving care for victims: a study protocol of the evaluation of a centre for sexual and family violence. BMJ Open 2016;6: e011545. doi:10.1136/ bmjopen-2016-011545

- Prepublication history for this paper is available online. To view these files please visit the journal online (http://dx.doi.org/10.1136/ bmjopen-2016-011545).

Received 16 February 2016 Revised 30 May 2016 Accepted 8 July 2016

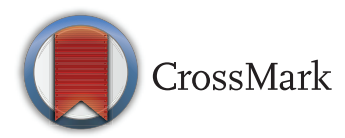

${ }^{1}$ Department of Primary and Community Care, Gender \& Women's Health, Radboud University Nijmegen Medical Center, Nijmegen, The Netherlands

2Department of Clinical Psychology, Behavioural Science Institute Radboud University Nijmegen, Nijmegen, The Netherlands

Correspondence to Elza Zijlstra;

Elza.Zijlstra@radboudumc.nl

\section{ABSTRACT}

Introduction: Worldwide, sexual and family violence are highly prevalent problems. Victims of sexual and family violence often do not seek formal help in the acute phase. When they do seek help, they encounter a system of scattered care. For this reason, a centre for sexual and family violence was launched in Nijmegen, the Netherlands. The centre provides multidisciplinary care for victims of acute sexual and/or family violence. With the study described in this study protocol, we want to evaluate the implementation process and the reach of the Center for Sexual and Family Violence Nijmegen (CSFVN).

Methods and analysis: We will conduct a mixedmethods study including quantitative and qualitative methods of data collection and analysis. Data about the implementation process will be obtained via semistructured interviews and focus group discussions. Content analysis will be done in software program Atlas.ti. Analysis of file data will be undertaken to assess the reach of the CSFVN (patient characteristics and characteristics of the care they received). The data will be analysed in SPSS.

Ethics and dissemination: The Medical Ethics Committee of the Radboud University Nijmegen Medical Center approved the study protocol under file number 2012-1218. Dissemination will be done by submitting scientific articles to academic peer-reviewed journals. We will present the results at relevant international, national and local conferences and meetings. We will send press releases to relevant media. We will share the results with the network of assault centres in the Netherlands.

\section{INTRODUCTION}

Worldwide, the prevalence of sexual and family violence is high as about one in three women have experienced intimate partner violence or non-partner sexual violence. ${ }^{1}$ A survey in the USA showed that $14 \%$ of the men had suffered from severe partner violence and about a quarter had experienced sexual violence-the perpetrator mostly being a man. ${ }^{2}$ The Netherlands is not an exception when it comes to high prevalence

\section{Strengths and limitations of this study}

- This study will add to the scarce amount of studies performed in assault centres by giving insight into the implementation process as well as the reach of an assault centre.

- This study uses a mixed-method design which combines quantitative and qualitative research methods. As a result of a process of triangulation, we will give a comprehensive view on the functioning of an assault centre.

- Dealing with a vulnerable target group might result in a limited recruitment of participants. Therefore, we possibly have to adapt our research methods. Furthermore, owing to the specific situation of our centre, we have to be cautious to generalise.

rates. A cross-sectional survey with face-to-face interviews showed that $45 \%$ of the population had experienced some form of family violence. ${ }^{3}$ As for rape, $12 \%$ of the Dutch women reported having been raped. For men, the percentage is $3 \% .^{4}$ The negative consequences for the victims' health are numerous. Physical problems are, for example, sexually transmitted diseases (STDs), unwanted pregnancies and pregnancy complications and chronic pain. Post-traumatic stress disorder (PTSD), depression, anxiety disorders and substance abuse are examples of psychological consequences. ${ }^{1} 56$ Healthcare usage rates, therefore, are high and the costs to society are considerable. ${ }^{1}$

Victims of sexual and family violence often do not seek formal help in the acute phase. ${ }^{78}$ Shame and fear play an important role in help-seeking behaviour. Moreover, victims often consider the violence as a private matter or as 'not serious enough'. Furthermore, they are afraid that they will not be believed or think that formal services cannot help. ${ }^{7-12}$ When victims do seek help, they encounter a system of scattered care. Multidisciplinary collaboration between 
organisations is poor. ${ }^{10}{ }^{13}$ Fragmentation of care leads to suboptimal care and higher costs. ${ }^{14}$ Besides, victims might be treated negatively by the police and/or healthcare professionals and do not always receive the medical care they need, such as pregnancy counselling or safety assessment. ${ }^{13}{ }^{15} \quad 16$ A comprehensive and coordinated system response to violence is needed. ${ }^{17}$ Multidisciplinary care, preferably in an assault centre, has been recommended by several researchers in the field of sexual and family violence. ${ }^{10}{ }^{18-20}$ Worldwide, sexual assault centres (SACs) or sexual assault response teams (SARTs) have been established. In 2012, the Center for Sexual and Family Violence Nijmegen (CSFVN) was launched-the first Dutch assault centre that combined care for victims of acute sexual and family violence. The target population of the CSFVN consists of female and male victims of all ages who are a victim of acute sexual and/or family violence. The CSFVN explicitly decided to give care to victims of sexual and family violence because both are forms of gender-based violence and share causes, risk factors and health consequences. ${ }^{21}$ Both require a sensitive, respectful, integrative approach by caregivers. ${ }^{17}$ Furthermore, many acts of sexual violence are committed by an (ex) partner or a family member. ${ }^{12} 2223$ The CSFVN wants to improve care for victims by providing multidisciplinary acute care and adequate follow-up care. Medical, forensic and police services provide the acute care. A case manager provides the follow-up care. The case manager keeps in contact with the victim's general practitioner (GP). The CSFVN thinks it is important to actively involve GPs by contacting them after the acute care, providing information about the CSFVN and giving training about violence. The primary healthcare system, especially the GP, is an important entry point for victims of family violence to seek help, and GPs can play an important role in proper referral and adequate follow-up care. ${ }^{24-27}$ GPs often have a long-term relationship with their patients and are easy accessible. This leads to continuity of care and a strong biopsychosocial, patient-centred focus. Since violence is hardly ever an isolated problem, this integrative view is important in giving adequate care.

\section{Current study}

SACs and SARTs seem to have promising outcomes on relationships among collaboration partners, help-seeking behaviour of victims and slightly on judicial outcomes. ${ }^{10}$ However, on an implementation level, assault centres face many challenges. A review of Greeson and Campbell $^{10}$ revealed organisational barriers, such as struggles to maintain adequate staffing for medical programmes, and problems with acquiring broad-based participation. Other barriers were conflicting goals, role confusion, role conflict and different views on confidentiality restrictions. However, studies on SACs and SARTs are scarce. It is important to pay attention to the process of implementation, next to the reach of assault centres. ${ }^{13}$ The aim of this study therefore is to evaluate the CSFVN by studying both its implementation process and its reach. We will conduct a mixed-method study design with quantitative and qualitative data collection and analysis. We will triangulate these data to evaluate in which way and to what extent the aims of the CSFVN have been reached.

To operationalise the evaluation, we formulated the following research questions: (1) what are the factors that facilitate or impede the implementation of the CSFVN? (2) what is the reach of the CSFVN measured by patient, assault and care characteristics? Until now, there are no extensive studies that evaluate the implementation and reach of a particular assault centre, and neither have studies integrated quantitative and qualitative data and the views of professionals and patients. Research is needed to evaluate effects and to apply improvements in the care provided. ${ }^{10} 1728$

\section{METHODS AND ANALYSIS}

Intervention: CAFVN

In October 2011, 1 year before the opening of the CSFVN, the head and the coordinator of the CSFVN made an inventory of bottlenecks in the current care for victims and the benefits of a CSFVN. Meetings were arranged with a broad range of services in the region. Agreements were made between collaboration partners. This led to the CSFVN whose structure and protocol we will now describe.

\section{Structure of the CSFVN}

We will describe the CSFVN according to the organisational levels that are used in American studies about SARTs. $^{29}$

\section{Context}

The CSFVN is located in Nijmegen, a town of more than 170000 people. Nijmegen is the biggest town in a largely rural area that is served by the CSFVN. The CSFVN will be part of a growing network of assault centres throughout the Netherlands.

\section{Goals}

The overall goal of the CSFVN is to improve the quality of care for victims of sexual and family violence. This goal is subdivided into three separate goals that contribute to reaching the overall goal: (1) improve helpseeking experiences of victims; (2) improve knowledge, attitude and behaviour of professionals towards victims of violence; and (3) improve interdisciplinary collaboration. These goals were formulated together by the members and have been recorded in a memorandum of understanding. The CSFVN executes the goals by offering multidisciplinary care at one location, building up expertise and supporting caregivers in giving help to assault victims. 


\section{Membership}

The CSFVN is a collaboration between the emergency department (ED) of the Radboud University Medical Centre, the District Police Department, the Community Health Services and an academic Primary Health Care Center. The academic Primary Health Centre employs GPs and has a strong collaboration with psychologists with expertise in PTSD. For research purposes, the health centre works closely together with the Department of Primary and Community Care/Gender and Women's Health. Apart from these core members, the CSFVN has contact with a broad range of caregivers, for example, victims' advocates, women's shelters and child protection services.

\section{Formal structures and leadership}

The steering group, which consists of representatives of the collaborating institutions, has quarterly meetings to monitor the quality of care. The meetings are organised by the coordinator of the CSFVN and led by the formal head of the CSFVN. The coordinator and head are not sexual assault responders themselves.

\section{Collaborative activities}

The CSFVN organises two-monthly multidisciplinary meetings in which cases are reviewed, bilateral meetings with stakeholders and educational meetings for care providers in the region. Furthermore, there are two-weekly meetings between the coordinator and the head of the CSFVN and the case manager. The CSFVN makes use of a protocol which is annually reviewed by the collaborative partners. It has a memorandum of understanding in which agreements are written down on aims, tasks, responsibilities, exchange of patient data and finances. The memorandum of understanding is signed by authorised representatives of the collaborating organisations. The CSFVN publishes an annual evaluation report online with (limited) patient data (eg, gender, age groups, type of assault, perpetrator characteristics), conducted activities (eg, education and research) and changes in the working process.

\section{Cross-system coordination}

Appointments have been made for cross-system coordination. They are noted in a protocol. These appointments make sure that the police, forensic physician, ED staff and the case manager work closely together and involve each other in each other's work.

\section{Protocol of the CSFVN}

The CSFVN offers medical care and contact with the police and forensic physician to victims of acute sexual (within 7 days after the incident) and family violence. The services of the CSFVN take place at the ED. The protocol consists of the following steps carried out by the ED staff: (1) provide acute medical care; (2) counsel, test and treat STDs including hepatitis B and HIV; (3) counsel on pregnancy and take pregnancy measures if necessary; (4) inform the patient about legal steps and contact the police at the patient's request. If the patient wishes to report, the police comes to the ED to give information about reporting. The forensic physician is called in by the police when the victim considers to report and performs a medical forensic examination; (5) assess the patient's safety and that of her/his children; (6) inform the patient about follow-up care via the CSFVN; (7) send a letter to the patient's GP. If necessary, medical specialists are called in (paediatrician, gynaecologist, surgeon or infectious disease specialist).

If there are no medical or safety reasons to keep the patient in the hospital, the patient is subsequently sent home. One day later, the CSFVN case manager will phone the patient to invite her or him for a consultation at the academic Primary Health Care Center. The main aims of this consultation are to give emotional and practical support, to provide psychoeducation and to refer to proper follow-up care. The case manager screens for PTSD at 1 month and 3 months after the incident as well.

\section{Study design}

Since the CSFVN is an innovative, complex and new intervention and research on assault centres is scarce, especially in Europe, we will use a pragmatic instead of a theoretical approach for our evaluation. We will conduct a mixed-method study design with quantitative and qualitative data collection and analysis. We will triangulate these data to evaluate in which way and to what extent the aims of the CSFVN have been reached. Mixed-method studies are suitable for exploratory research and for capturing the process and circumstances under which a particular intervention takes place by making use of triangulation of data. ${ }^{13}$ We decided per research question which research method fitted the question the best. Qualitative research methods, namely focus group discussions and semistructured interviews, will be used to explore the facilitating and constraining factors in the implementation. Quantitative research methods in the form of file analysis will be used to assess the characteristics of our patient population and the care they received. We will use the consolidated criteria for reporting qualitative research (COREQ) criteria for designing and reporting the qualitative research. ${ }^{30}$ The COREQ is a 32-item checklist for reporting qualitative research on the following fields: research team and reflexity, study design and analysis and findings. Data will be collected and analysed from January 2013 up to and including June 2016 (31/2 years after the start of CSFVN).

\section{Participants}

\section{Victims of sexual and family violence}

All patients who received care of the CSFVN will be included in the database that the CSFVN developed. The database will be used for the quantitative outcomes. For the qualitative outcomes, all patients aged 16 years 
and older who received follow-up care via the case manager will be approached for a short interview about their experiences with the CSFVN. Victims below the age of 16 years or intellectual disabled victims will not be interviewed themselves, but we will interview their parents or caregivers when they have accompanied the victim to the CSFVN. For the victims aged 16 and 17 years, we will have to obtain both the patient's as both of his/her parents or caregivers informed consent.

Four victims will be selected according to the purposive sampling technique for extensive semistructured interviews. We think four interviews of 1 hour each will be sufficient to add in-depth information to the short interviews about the experiences of victims with the CSFVN. If four interviews are not sufficient, we will expand the number of interviews. In the sample of four participants, we include victims of sexual and family violence of different age groups and gender. We will choose the participants after having analysed the database. For example, when we notice that the CSFVN is visited mainly by victims in the age group $18-25$ years who have been raped by a known perpetrator, we will make sure we will interview one victim with this profile. Victims will be chosen in consultation with the case manager on their suitability (eg, a victim must have a certain capability to reflect on the care of the CSFVN and must not be too vulnerable).

\section{Collaborating partners within the CSFVN}

The participants will be representatives of the collaborating partners of the CSFVN, namely the ED, the Community Health Services, the police and the academic Primary Health Care Center. We will interview policymakers as well as direct (health) care providers.

\section{Measures}

\section{Implementation process}

Information will be obtained by conducting semistructured interviews and focus group discussions with the collaborating partners, care providers and the patients of the CSFVN. Interview guides will be composed on the basis of existing literature and discussions with the supervising committee (GH, ALJ, SLFW). In the short interviews with victims, we will ask about their experiences with the medical, legal and follow-up services. The results of the short interviews are used to compose the interview guide for the extensive interviews with victims in order to clarify results and gather in-depth information. As for the professionals, we will ask about their experiences with the CSFVN at the individual (professional) level and at the level of interdisciplinary collaboration. We will focus on facilitating and constraining factors in the implementation. We consider factors in the implementation process as facilitating when they contribute to reaching the aims of the CSFVN: improve help-seeking experiences of victims, improve the knowledge, attitude and behaviour of professionals and improve interdisciplinary collaboration. Constraining factors can be seen as challenges in reaching these aims. We will analyse our data at three levels: (1) the level of the victim, the level of the professional and the level of interdisciplinary collaboration. In our analysis, the victim will be put up front, since, in line with the WHO guidelines on responding to intimate partners' violence and sexual violence against women, ${ }^{31}$ we support a victim-centred approach.

\section{Reach of the CSFVN}

The reach of the CSFVN will be measured by analysing the CSFVN database that contains patient characteristics, such as: sociodemographic characteristics and use of medication; assault characteristics, such as perpetrator characteristics and use of alcohol and/or drugs; and care characteristics, such as received medical care and involvement of police and forensic physicians. To assess for PTSD, we will use the translated and validated Trauma Screening Questionnaire. ${ }^{32}$

\section{Data collection}

The stakeholders of the CSFVN who will be interviewed receive written information on the study and will be asked by email to participate. The interviewer is a researcher trained in interview techniques. The patients who will be interviewed are approached by phone. The case manager will conduct the short interviews with patients, since we think the case manager is more easily trusted and less threatening than a third person. The CSFVN has two case managers and to counteract bias, the patient will not be interviewed by the case manager who provided care. The extensive interviews with victims will be conducted by a trained interviewer who has experience with vulnerable populations. All interviews will be recorded and transcribed verbatim.

The CSFVN database consists of data obtained from medical, police, forensic and follow-up care files of the patients with CSFVN. The data will be made anonymous.

\section{Data analysis}

The qualitative data about the implementation of the CSFVN will be analysed by content analysis using the Atlas.ti software program. The data will be analysed by two researchers separately (EZ and a medical research trainee) in a process of open, axial and selective coding. The stakeholders who participate are given the chance to give feedback on their transcript before analysis.

We will use descriptive statistics to analyse the quantitative data from the patient files. SPSS will be used for the analysis. We will compose profiles of the victims who visit the CSFVN according to their sociodemographic characteristics, for example, age group and gender, and assault characteristics (eg, use of alcohol/drugs, known or unknown perpetrator). We will subsequently examine in what way different groups make use of the CSFVN, for example, by whom and in which time frame are they referred, do they report to the police and do they make use of the follow-up care? 


\section{ETHICS AND DISSEMINATION \\ Ethics}

The Ministry of Justice gave consent for conducting analysis of police and forensic files on 9 September 2015 (file number PaGlBIZl48362).

The collaborating partners and the care providers of the CSFVN will receive written and oral information on the study. This information will contain, for example, information on the voluntary character of participation, the possibility to withdraw at any moment and the opportunity to ask questions of a researcher with no links to the conductors of this study. They have more than a week to consider whether they want to participate or not. Before the interview starts, the participants will be asked to sign an informed consent form. In the articles that will be based on this interview, we will make sure that quotes are not convertible to a particular person.

We are dealing with a vulnerable target group. Our database will therefore be made fully anonymous. The database is saved at an extension at the computer to which only EZ has access. The code list will be stored in a secure data storage archive. The case manager will give an opportunity to victims to conduct the interview by telephone. This will make the burden for the victims lower than if they were to visit the centre. Information on the study will be sent to their address or will be relayed over the phone-depending on the choice of the victim. We will make clear appointments with the victims on when and how long to call-bearing their safety in mind. In the articles that will be based on this interview, we will make sure that particular persons cannot be traced.

\section{Dissemination}

Dissemination will be done by submitting scientific articles to academic peer-reviewed journals. We will present the results at relevant international, national and local conferences and meetings. We will send press releases to relevant media. We will share the results with the network of assault centres in the Netherlands.

\section{DISCUSSION}

Sexual and family violence are problems that have considerable negative consequences for the victim's health. Victims who seek help encounter a system of scattered care. Since low-threshold multidisciplinary care is urgently needed, the CSFVN has been launched in 2012. The CSFVN is a multidisciplinary collaboration between medical, psychosocial and judicial services. A victim of sexual and/or family violence receives multidisciplinary care in the acute setting and follow-up care in the primary healthcare context. This study will evaluate the implementation process and the reach of the CSFVN. We expect that an assault centre improves helpseeking experiences of victims and enhances interdisciplinary collaboration when implemented well. Literature is scarce and proper research is needed to rectify the existence of assault centres. We want to contribute to this 'pioneer work'. This information will be useful for parties who intend to set up an assault centre or a multidisciplinary collaboration programme for violence and it helps to improve the care of existing assault centres.

\section{Strength and limitations}

This study is the first study in the Netherlands that focuses on the evaluation of an assault centre at both the implementation and outcome levels. In Europe, studies have been done about assault centres, but they do not deal explicitly with the implementation processes. Qualitative data are lacking. We will use a mixed-methods study, which uses different sources and combines quantitative and qualitative research methods. This mixed-methods study is recommended by researchers in the field of SACs and SARTs. ${ }^{13}{ }^{28}$ Triangulation of data will enable us to give a broad and extended view on the functioning of an assault centre.

The centre will be studied from its early preparations for the start (2011) until 3 years after the opening, which is expected to give us a better understanding of its development in time. We will involve a wide variety of participants with backgrounds in medical, psychosocial and/or legal disciplines. We explicitly want to involve our patients as well. Without the view of our target group, this evaluation would not be complete. However, involving the victims can become challenging. ${ }^{33}$ The victims went through traumatic events they want to forget. We have to take into account that the burden of our evaluation should be as low as possible. We will possibly lose a certain number of patients to follow-up.

Since this study will be performed in one assault centre-the CSFVN-which has its particular aims and philosophy and is bound to local circumstances, conclusions should be extrapolated to other settings with caution. Also, since this is an exploratory study, we are aware that some findings will ask for more in-depth research. Therefore, we think that our results should serve as a basis for subsequent and ongoing research.

Contributors ALJ and EZ designed the study and drafted the paper. TL, SL and $\mathrm{GH}$ reviewed and advised on the paper. EZ will perform the study. TL will supervise the study. All authors approved on the final version of the paper.

\section{Competing interests None declared}

Ethics approval The Medical Ethics Committee of the Radboud University Nijmegen Medical Center decided that the study was in accordance with the applicable rules concerning the review of research ethics committees and informed consent (file number 2012-1218).

Provenance and peer review Not commissioned; externally peer reviewed.

Data sharing statement The submitted paper is not an original research article, but a study protocol. There are no research data involved as yet.

Open Access This is an Open Access article distributed in accordance with the Creative Commons Attribution Non Commercial (CC BY-NC 4.0) license, which permits others to distribute, remix, adapt, build upon this work noncommercially, and license their derivative works on different terms, provided the original work is properly cited and the use is non-commercial. See: http:// creativecommons.org/licenses/by-nc/4.0/ 


\section{REFERENCES}

1. World Health Organization. Global and regional estimates of violence against women: prevalence and health effects of intimate partner violence and nonpartner sexual violence. Geneva, World Health Organization, 2013.

2. Breiding MJ, Smith SG, Basile KC, et al. Prevalence and characteristics of sexual violence, stalking, and intimate partner violence victimization-National Intimate Partner and Sexual Violence Survey, United States, 2011. MMWR Surveill Summ 2014;63:1-18.

3. Van Dijk T, Flight S, Oppenhuis E, et al. Domestic violence: a national study of the nature, size and effects of domestic violence in the Netherlands. Eur J Crim Pol Res 1998;6:7-35.

4. De Haas S, van Berlo W, Bakker F, et al. Prevalence and characteristics of sexual violence in the Netherlands, the risk of revictimization and pregnancy: results from a national population survey. Violence Vict 2012;27:592-608.

5. Campbell JC. Health consequences of intimate partner violence. Lancet 2002;359:1331-6.

6. Cavanaugh CE, Martins SS, Petras $\mathrm{H}$, et al. Mental disorders associated with subpopulations of women affected by violence and abuse. J Trauma Stress 2013;26:459-66.

7. European Union Agency for Fundamental Rights. Violence against women: an EU wide survey. European Union Agency for Fundamental Rights, 2014. http://fra.europa.eu/sites/default/files/ fra-2014-vaw-survey-main-results-apr14_en.pdf (accesssed 15 Feb 2016)

8. Wolitzky-Taylor KB, Resnick HS, Amstadter AB, et al. Reporting rape in a national sample of college women. J Am Coll Health 2011;59:582-7

9. Shea CA, Mahoney M, Lacey JM. Breaking through the barriers to domestic violence intervention. Am J Nurs 1997;97:26-33.

10. Greeson MR, Campbell R. Sexual assault response teams (SARTs) an empirical review of their effectiveness and challenges to successful implementation. Trauma Violence Abuse 2013;14:83-95.

11. Patterson D, Greeson M, Campbell R. Understanding rape survivors decisions not to seek help from formal social systems. Health Soc Work 2009;34:127-36.

12. Fanslow JL, Robinson EM. Help-seeking behaviors and reasons for help seeking reported by a representative sample of women victims of intimate partner violence in New Zealand. J Interpers Violence 2010;25:929-51.

13. Campbell R, Patterson D, Bybee D. Using mixed methods to evaluate a community intervention for sexual assault survivors: a methodological tale. Violence Against Women 2011;17:376-88.

14. Kodner DL. All together now: a conceptual exploration of integrated care. Healthc Q 2009;13 Spec No:6-15.

15. Campbell R. The psychological impact of rape victims. Am Psychol 2008;63:702.

16. Rhodes KV, Kothari CL, Dichter M, et al. Intimate partner violence identification and response: time for a change in strategy. J Gen Intern Med 2011;26:894-9.
17. García-Moreno C, Hegarty K, d'Oliveira AFL, et al. The health-systems response to violence against women. Lancet 2015;385:1567-79.

18. Ensink B, Van Berlo W. Indringende herinneringen. De ontwikkeling van klachten na een verkrachting (Intrusive memories: development of psychological problems after sexual assault). Utrecht: NISSO/ Delft: Eburon, 1999.

19. Bari S, Jawad R. Management of sexual assault and the importance of Sexual Assault Referral Centres (SARCs). J Fam Plann Reprod Health Care. 2010;36:115-16.

20. Eogan M, McHugh A, Holohan M. The role of the sexual assault centre. Best Pract Res Clin Obstet Gynaecol 2013;27:47-58.

21. Hamby S. Intimate partner and sexual violence research: scientific progress, scientific challenges, and gender. Trauma Violence Abuse 2014;15:149-58.

22. Finkelhor D, Dziuba-Leatherman J. Children as victims of violence: a national survey. Pediatrics 1994;94:413-20.

23. Russell DE. The incidence and prevalence of intrafamilial and extrafamilial sexual abuse of female children. Child Abuse Negl 1983;7:133-46.

24. Malpass A, Sales K, Johnson M, et al. Women's experiences of referral to a domestic violence advocate in UK primary care settings: a service-user collaborative study. Br J Gen Pract 2014;64: e151-e8.

25. Kennedy KM, White C. What can GPs do for adult patients disclosing recent sexual violence? Br J Gen Pract 2015; 65:42-4.

26. Feder $\mathrm{G}$. Responding to intimate partner violence: what role for general practice? Br J Gen Pract 2006;56:243-4.

27. Mol SS, Arntz A, Metsemakers JF, et al. Symptoms of post-traumatic stress disorder after non-traumatic events: evidence from an open population study. Br J Psychiatry 2005;186:494-9.

28. Moylan CA, Lindhorst T, Tajima EA. Sexual Assault Response Teams (SARTs) mapping a research agenda that incorporates an organizational perspective. Violence Against Women 2015;21:516-34.

29. Greeson MR, Campbell R. Coordinated community efforts to respond to sexual assault a national study of sexual assault response team implementation. J Interpers Violence 2014;30:2470-87.

30. Tong A, Sainsbury $P$, Craig J. Consolidated criteria for reporting qualitative research (COREQ): a 32-item checklist for interviews and focus groups. Int J Qual Health Care 2007;19:349-57.

31. World Health Organization. Responding to intimate partner violence and exualviolence against women: WHO clinical and policy guidelines. Geneva, World Health Organization, 2013.

32. Brewin CR, Rose S, Andrews B, et al. Brief screening instrument for post-traumatic stress disorder. Br J Psychiatry 2002;181: 158-62.

33. Herbert CP, Grams GD, Berkowitz J. Sexual assault tracking study: who gets lost to follow-up? CMAJ 1992;147:1177. 